\title{
Application of evolutionary algorithms to optimize cooling channels
}

\author{
Narasimha R. Nagaiah ${ }^{*}$ and Christopher D. Geiger ${ }^{2}$ \\ ${ }^{1}$ University of Central Florida, 32816 Orlando, Florida, USA \\ ${ }^{2}$ Universal Orlando Resort, 32819 Orlando, Florida, USA
}

Received: 16 September 2018 / Accepted: 24 February 2019

\begin{abstract}
The design and development is a complex, repetitive, and more often difficult task, as design tasks comprising of restraining and conflicting relationships among design variables with more than one design objectives. Conventional methods for solving more than one objective optimization problems is to build one composite function by scalarizing the multiple objective functions into a single objective function with one solution. But, the disadvantages of conventional methods inspired scientists and engineers to look for different methods that result in more than one design solutions, also known as Pareto optimal solutions instead of one single solution. Furthermore, these methods not only involved in the optimization of more than one objectives concurrently but also optimize the objectives which are conflicting in nature, where optimizing one or more objective affects the outcome of other objectives negatively. This study demonstrates a nature-based and bioinspired evolutionary simulation method that addresses the disadvantages of current methods in the application of design optimization. As an example, in this research, we chose to optimize the periodic segment of the cooling passage of an industrial gas turbine blade comprising of ribs (also known as turbulators) to enhance the cooling effectiveness. The outlined design optimization method provides a set of tradeoff designs to pick from depending on designer requirements.
\end{abstract}

Keywords: Multi-objective optimization / Numerical simulation / Genetic algorithm / Evolutionary algorithm / Heat transfer / Fluid dynamics / Gas turbine / Blade / Internal cooling channel

\section{Introduction}

The field of mechanical design is the one segment of the industry that is not evolved the way other segments of the industry evolved, for example, the materials innovation and computer technology evolved significantly in fastpaced than the mechanical design methods. Today, the mechanical system and component design is still an awfully slow process and takes an enormous amount of labor and cost-prohibitive steps of iterations. In the sense, there is no known method of autonomous feedback based design process in place to design and optimization of a component iteratively without human intervention. In the last two decades, the advancement in computational power introduced various mechanical simulation and design tools to help ease the pain of mechanical component designers. Now, numerical simulation tools and traditional experimental techniques become an essential component of the mechanical part design and therefore are readily utilized to

\footnotetext{
* e-mail: narasimha.nagaiah@ucf .edu
}

identify and affirm numerous design options. However, the main drawback of using experimental and simulation tools is they are extremely time consuming, labor intense and frequently cost-prohibitive. Additionally, these methods fail to address the interactions of design variables and objectives under consideration and their effects on various physical and performance factors. The present conventional designing methods do not address to satisfy multiple and frequently contradictory design variables and objectives which greatly influence the operation and price of the part.

To address above shortcomings of the conventional simulation and experimental design methods, the author first proposed multi-objective optimization framework integrated with commercial numerical simulation tool and nature-inspired evolutionary based genetic algorithm (GA) to solve for two conflicting objective functions [1]. In this research article, the author introduced the third conflicting objective function, iteratively selected suitable genetic algorithm operators values (i.e., crossover and mutation probability) and shown the effect of introducing more design variables while optimizing the design of the complex 


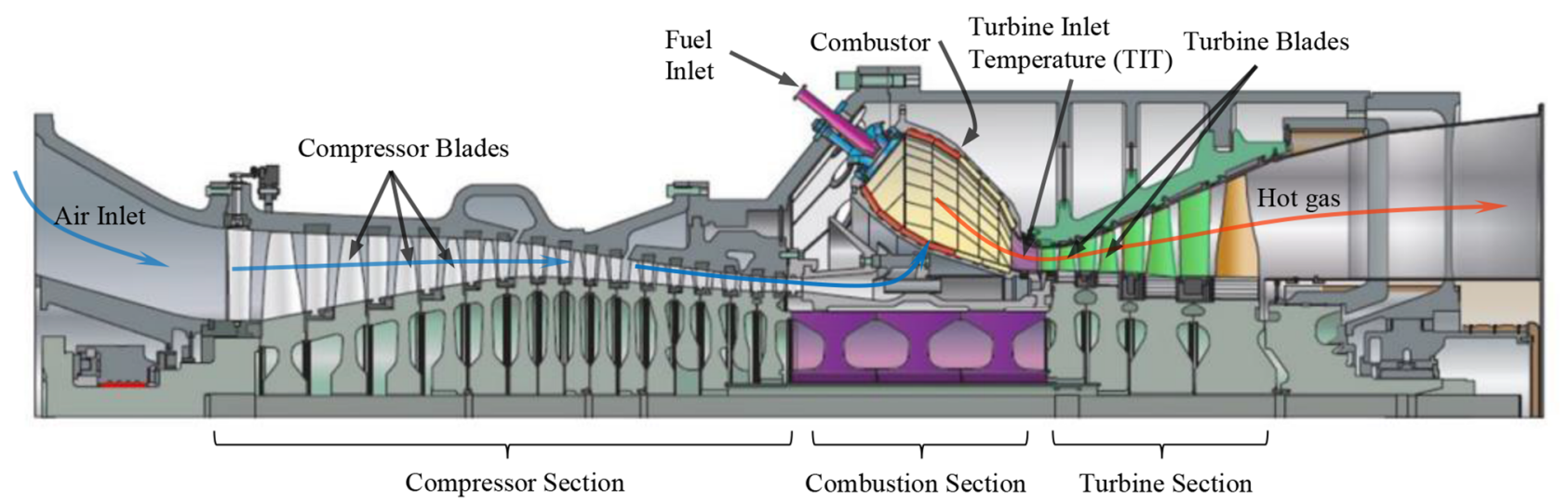

Fig. 1. A sectional view of the in-line axial gas turbine engine (courtesy of Siemens).

mechanical component. An industrial gas turbine blade internal cooling passage design is selected as the test program for the study of the suggested framework. Because of the necessity of high operating temperature to obtain high power output and harsh environment the turbine blades demand most efficient cooling methods to prolong the life span of the highly expensive blades and in turn avoid power outage.

The proposed framework is introduced and tested by selecting six design variables and three design objectives, where all design variables directly affect the selected objectives. The three objectives selected in this research are: - heat transfer coefficient $(h)$ of the blade cooling passage, which will be the indication of amount of heat transfer to improve the cooling and the aim here is to maximize $h$ value to improve the blade life expectancy and reliability of the turbine;

- coolant pressure drop $(\Delta \mathrm{p})$ in the cooling channel, where the aim here is to minimize the pressure drop to sustain the continuous flow of the coolant;

- geometric specification of blade cooling passage, especially the cavity area of $(A)$ cooling passage, where the aim here is to maximize this objective.

The increase in the blade cooling passage cavity area results in minimization of the expensive material used for the blade fabrication. The objective functions selected are conflicting and influence the effectiveness of cooling of the blade and the amount of material used in its development. The computational simulation results obtained reveal the new optimization framework introduced is capable of generating, evaluating, and identifying hundreds and thousands of tradeoff design solutions in a fraction of time compared to it might take using the conventional experimental and simulation applications utilized for mechanical design optimization. This framework is a vital step past the present design optimization methods in the field of complex mechanical system and components design optimization.

\section{Design problem selection}

To show the feasibility and optimization method followed in the proposed framework, an industrial gas turbine blade cooling channel design is chosen. For many decades, gas turbines are known as energy workhorses and are in the center of nearly all electric power generating systems of the world. They are also widely utilized in aviation, space, sea transportation along with many more industrial applications. The sectional view of Figure 1 below shows the simplest form of gas turbine which is called an in-line axial flow turbine. It operates similar to a well-known internal combustion engine by burning fuel and compressed air combinations. The combusted hot gas combination of fuel, air and unburned hydrocarbons can attain ultra-high temperatures as high as $1700^{\circ} \mathrm{C}$ and create significant pressure variations $[2,3]$. Ultra-high temperature gas from combustor with higher than metal melting temperature enters turbine stages, where each turbine stage contains set of vanes and blades installed on the stator (stagnant) and rotor (moving) discs respectively. At each one of these stages, the combusted and compressed hot gas expands and in turn runs the turbine to create rotational shaft power. This mechanical rotational power is partially utilized to drive the compressor, and the rest is used to run the generator to produce power [4].

The component of our interest is 1ststage turbine blades (Fig. 1) which are subjected to melting temperature and also incorporates the complex design of the cooling system. To lessen the simulation and computational effort, we selected a periodic section of the blade-cooling channel. Furthermore, the section is converted into a twodimensional (2D) geometry with ribs (turbulators) on both the top and bottom walls, as shown in Figure 2. It is the geometric form and design specification of these ribs and their attachment to the walls should be optimized are directly affect the objective functions values. Further, the critical design variables selected to control the geometric shape of the ribs. The ribs 1 and 2 radii $\left(R_{1}\right.$ and $\left.R_{2}\right)$ and fillet radii $\left(R_{3}, R_{4}, R_{5}\right.$, and $\left.R_{6}\right)$ between ribs 1 and 2 and blade wall surface are treated as vital design variables. In cooling channels, ribs induce turbulent mixing by separation and reattachment of coolant flow to improve the heat transfer. It is observed that the heat transfer is very high in the reattachment locations, but it is significantly low at the coolant flow separation location as a result of ribs. The coolant flow separation and reattachment mechanism are all directly affected by the radii of the ribs. 


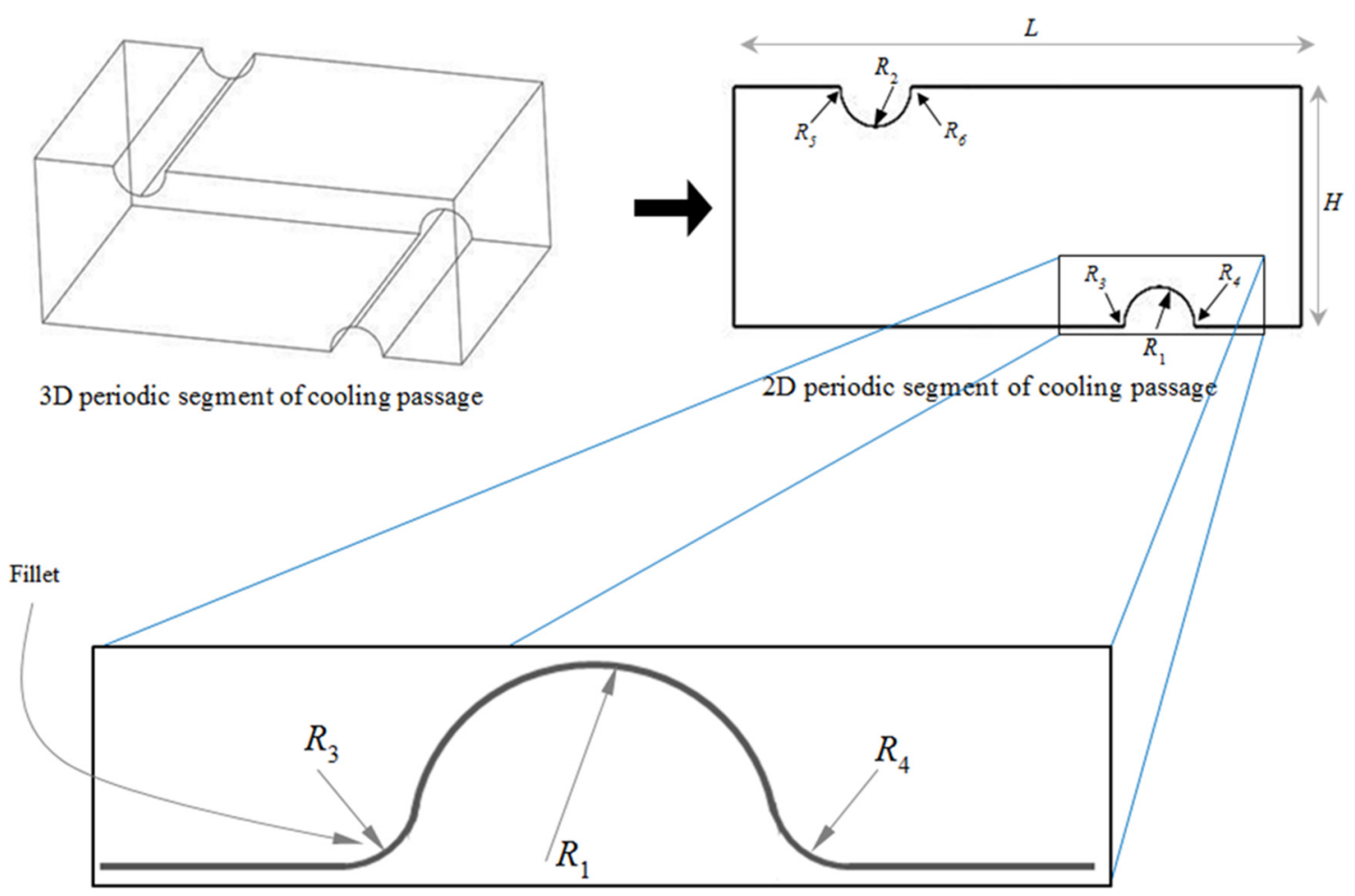

Fig. 2. Two-dimensional (2D) periodic section of the cooling channel and variables of interest.

The three objective functions considered, maximization of heat transfer coefficient $(h)$, minimization of coolant pressure drop $(\Delta \mathrm{p})$ and maximization of cooling passage cavity area of $(A)$, are all directly influenced by the ribs geometric specification. The variation in radii $R_{1}$ and $R_{2}$ increases/decreases the size of the ribs, turbulent mixing and surface area inside the cooling channel to enhance/ reduce the heat transfer from the blade to the coolant. However, the rib size increase causes the increase of coolant flow pressure drop and at the same time increases blade material usage. On the other hand, the fillet radii reduce pressure drop and improve heat transfer rate from blade to coolant by forming a smooth surface transition between blade wall and ribs (refer to Fig. 2 for an exploded view of blade's periodic segment of cooling passage). Therefore, varying these design variables may influence the heat transfer coefficient $h$, the coolant pressure drop $\Delta p$ and the material consumed by changing cavity area $A$.

Furthermore or more detailed description for the turbine cooling system and its significance, the selection of periodic segment from cooling channel, selection of design variables and genetic algorithm control parameters, refer to author's previous publications $[1,14]$.

\section{Literature review}

The conventional methods in the design optimization of the mechanical system or component in the engineering field are bound to the experimental and numerical simulations methods. Quite a bit of research was performed and presented with these conventional methods in the last few decades. In recent times, more and more researchers turned their research focus towards combinatorial and stochastic design optimization and identified a wide range of methods available to perform component design optimization using computers. These optimization approaches are drawing more attention and getting more popularity in engineering design problems due to the increased availability of readily affordable computers with higher computing power. In recent studies, it is evident that combinatorial and stochastic methods are broadly applied in engineering design problems in which the attention is either maximizing or minimizing a specific objective or set of objectives. These optimization methods covering various design problems in engineering have been developed over time, and they can be further widely categorized as shown in Figure 3.

But noticeably few worked on these optimization methods for components design and further even fewer utilized the concept of evolutionary techniques for multiple objectives optimization in a mechanical part or mechanical system design problems. The literature review here focuses on prior study linked to multi-objective optimization of blade cooling passage specification which is the subject of this study.

Over the past 50 years, there is a significant amount of research has been conducted related to turbine blade cooling methods. Scientists and engineers employed theoretical, computational simulation and experimental methods to design, optimize and enhance cooling techniques for the gas turbine blades. Recent publications focusing mainly on the gas turbine blade cooling technologies and heat transfer related to the cooling is 


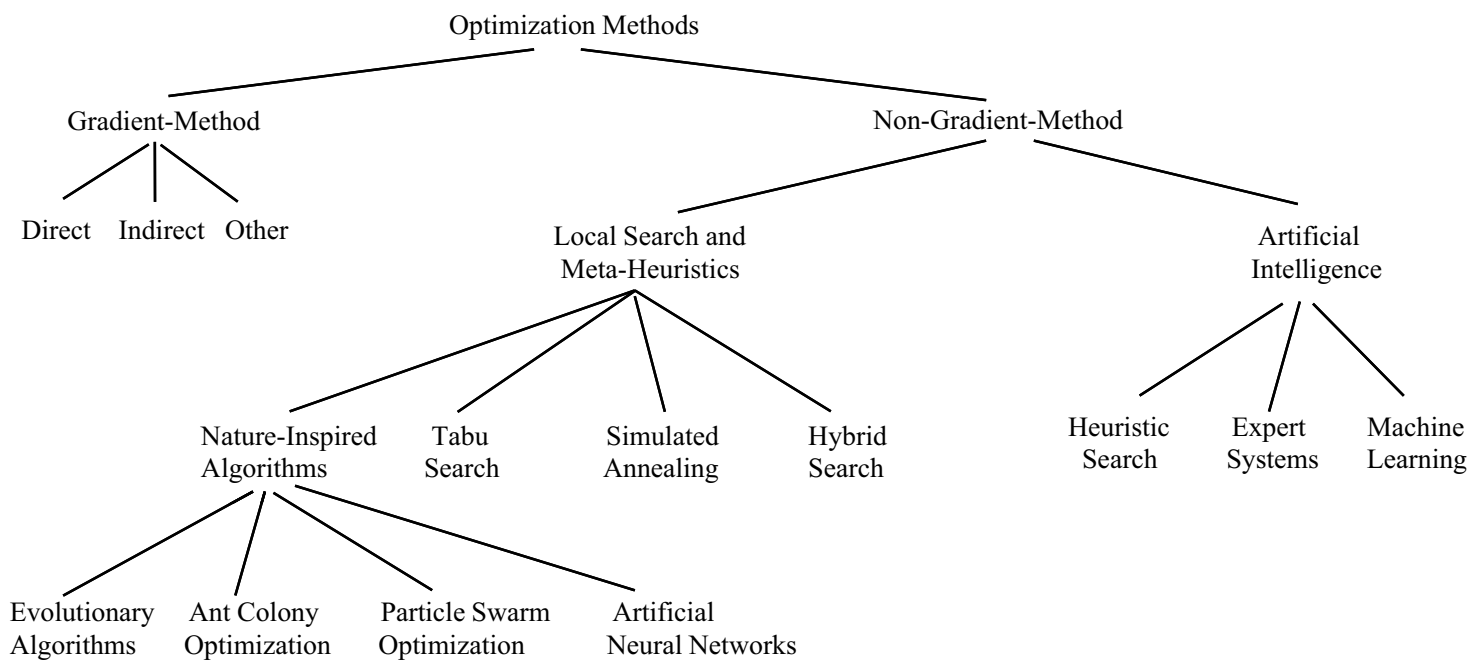

Fig. 3. Outline of optimization methods for engineering design problems.

offered by Han et al. [4,5], Logan [6] and Goldstein [7]. The usage of more than one objective or multiple objective optimizations in mechanical engineering, specifically in thermal-fluid design problems are comparatively new and have attracted attention recently. Specifically, in the past decade, we have observed a sharp rise in heat transfer and fluid dynamics related optimization with evolutionary algorithms (EAs). Gosselin et al. [8] published a comprehensive review of the use of the genetic algorithms, the most known representative of the family of EAs to solve multi-objective optimization problems in the area of heat transfer.

The well-established techniques to improve the cooling by enhancing heat transfer in a blade cooling air flow channel is to corrugate the internal walls with ribs (called turbulators), so the surface area increases and helps increase heat transfer from blade to cooling air and enhance overall cooling of the blade to maintain the temperature below the melting point. Researchers extensively studied various configurations of cooling channel ribs design in the gas turbine blade to improve the heat transfer and cooling effectiveness [9]. On the other hand, the insertion of rib turbulators creates other drawbacks like the drop in coolant's velocity and pressure. Therefore, it is ideal to apply design optimization techniques with an ability to solve a number of objectives concurrently. The adoption of multi-objective optimization to internal cooling channel design helps enhance the blade cooling and in turn its useful life, and also it could eventually be applied in another area of research in mechanical design optimization.

The design optimization of cooling channel design specification is broadly researched by Kim and Kim [10], who conducted the optimization of internal cooling channels with rectangular ribs [11], V-shaped ribs [12] with straight ribs design and the change in angle of the ribs [13]. They chose the values of design variables with the composite objective function described as a linear function of friction drag coefficient (pressure drop) and heat transfer coefficient. They further suggested that employing a numerical simulation strategy presents a dependable and economical means of designing and optimizing heat transfer surfaces. It is also vital to note that both objective functions considered within their research are the heat transfer coefficient $(h)$ and pressure drop $(\Delta p)$. On the other hand, the most important factor is these two objective functions are integrated to build a composite function with a single objective and to utilize the concept of the vector of weights. Selection of these approximated weights is entirely predicated on designer's expertise and discretion, which might lead to significant errors in design optimization when the weight factors are not chosen carefully.

In conclusion, because of the intricate design of cooling channel, and the type of fluid flow and heat transfer phenomena involved, only a few researchers tried to apply multi-objective design optimization methods to gas turbine blade cooling channel design. The limited research studies in this area considered two objective functions and combined them to form a composite function with a single objective. However, no present study concurrently took into account three different objectives separately and independently to optimize the design under consideration concurrently by giving significance to all objectives. The current study investigates a multi-objective design optimization technique by incorporating nature-inspired evolutionary algorithms and commercial simulation tool to discover a set of Pareto optimal solutions for the design of ribs in the blade's cooling channel.

\section{Multiple objective optimization framework}

The process of optimization is that solving the problems where the major criterion is to either minimize or maximize the objective function by choosing an arbitrary value of actual and/or integer decision variables (design variables) values inside the specified range. The process of deriving the best solution for a problem with just one objective 


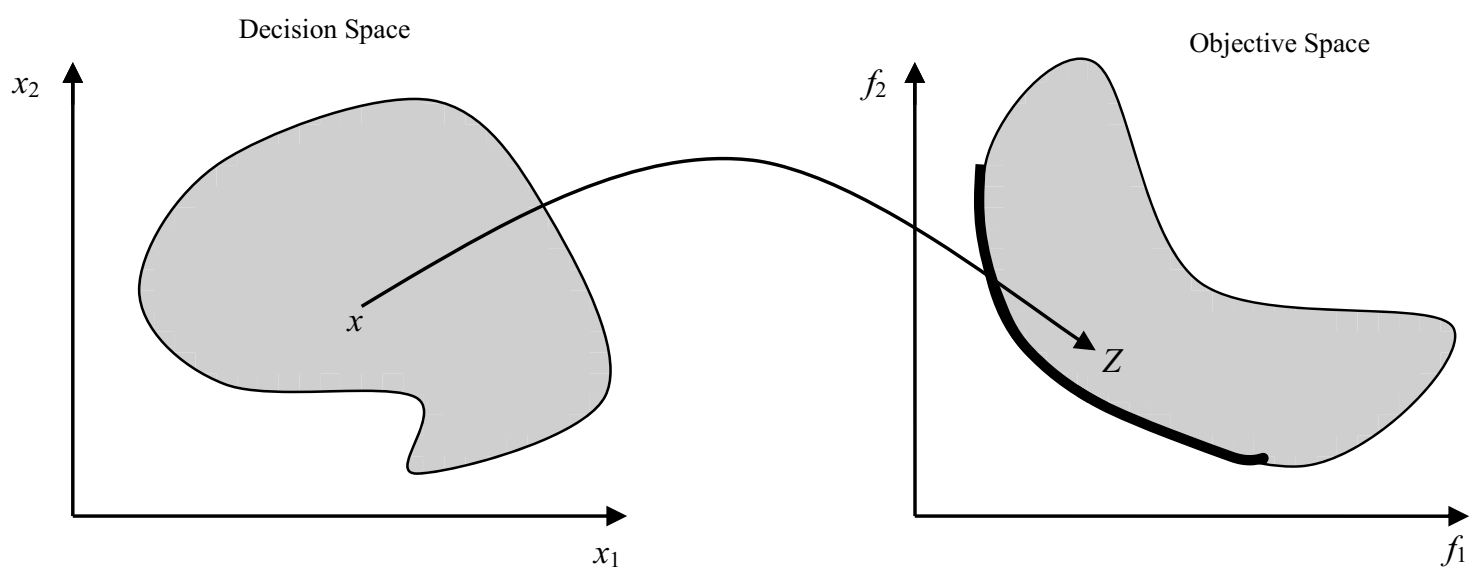

Fig. 4. Demonstration of the decision variable space and its corresponding objective space.

function is known as a single objective optimization problem. Nevertheless, in the real world, it is evident that problems involve multiple and conflicting objectives more often. A Multi-objective optimization problem (MOOPs) comprises more than one objective function. In such problems, if the objectives were chosen are in contradictory to each other, then there is no single optimal solution, instead set of optimal and compromise solutions (tradeoff solutions). A multi-objective optimization problem may be described as the following form:

$$
\min (\max ) \mathrm{f}(\mathrm{x}),
$$

at which $\mathbf{f}(\mathbf{x})$ is the vector of $m$ number of objective functions needs optimization, i.e., $\mathbf{f}(\mathbf{x})=\left(f_{1}(\mathbf{x}), f_{2}(\mathbf{x}), \ldots\right.$, $\left.f_{m}(\mathbf{x})\right)$, and solution $\mathbf{x}$ is a $n$-dimensional vector of decision variable values which are real or integer or both. Eq. (1), that is either converted to a minimization or maximization problem, usually subject to the following type of restrictions:

$$
\begin{gathered}
\mathrm{g}_{j}(\mathrm{x}) \leq \mathrm{b}_{j}, j=1,2, \ldots, k \\
a_{i} \leq x_{i} \leq \mathrm{b}_{i}, i=1,2, \ldots, n,
\end{gathered}
$$

where, $b$ is a $k$-dimensional vector of inequality constraints. Eq. (3) constrains the values of decision variable $x_{i}$ involving the lower $\left(a_{i}\right)$ and upper $\left(b_{\mathrm{i}}\right)$ bound. Similar to decision variable space, the objective functions will likewise compose a multidimensional space related to the decision variable space and is known as the objective space $Z$ (Fig. 4).

The main goal of this research study is to integrate evolutionary algorithms and numerical simulation technique and test the feasibility of the design optimization of the mechanical component with multiple conflicting objectives. The novel framework of multi-objective optimization is developed is illustrated in Figure 5 and it is applied to test its feasibility of optimization. The framework is divided into two main parts, consisting of an optimizer component and a simulation/evaluation component. The optimizer part repetitively produces multiple candidate designs by varying design variables value inside the user specified range. The numerical simulation perform the Evaluation of the candidate designs, and this part can be treated as a black box where:

- an input section that receives design variable values and transforms into new design to evaluate;

- an output section that evaluates the objective function values and transmits to the optimizer component.

In this study, a commercial code is used as the simulation component to carry out heat transfer and fluid dynamics simulation. Based on the candidate design evaluation results received from the simulation component, the optimizer creates the next set of design variable values through the perturbation for candidate designs. The iterative cycle continues until the designer prescribed termination criteria are achieved.

The design optimization of a gas turbine blade is further complicated by the addition of a cooling air system as shown in Figure 6. The main scope of this feasibility study of the proposed framework is to optimize the design of ribs (turbulators) to increase the internal surface area of the blade-cooling channel which is in direct contact with the coolant. The ribs increase the surface area and also promote turbulence to enhance the rate of heat transfer from the blade to the coolant. Figure 6 shows the internal cooling passage with rib-roughened walls, which is the focus of this multi-objective optimization study.

\subsection{Simulation/Evaluation component}

The simulator builds the computational model of the design and analyses the design to evaluate the objective function values, i.e., $h$ (heat transfer coefficient), $\Delta p$ (pressure drop) and $A$ (cooling passage cavity area) for a set of design variable values randomly chosen by the optimizer from the user-specified range of values. As described earlier, the radii $\left(R_{1}\right.$ and $\left.R_{2}\right)$ of ribs 1 and 2 , and fillet radii $\left(R_{3}, R_{4}, R_{5}\right.$, and $\left.R_{6}\right)$ are considered as design variables (Fig. 2). To solve for the objective functions for a particular rib design configuration (design variable values), the heat transfer problem is simulated and solved for the 


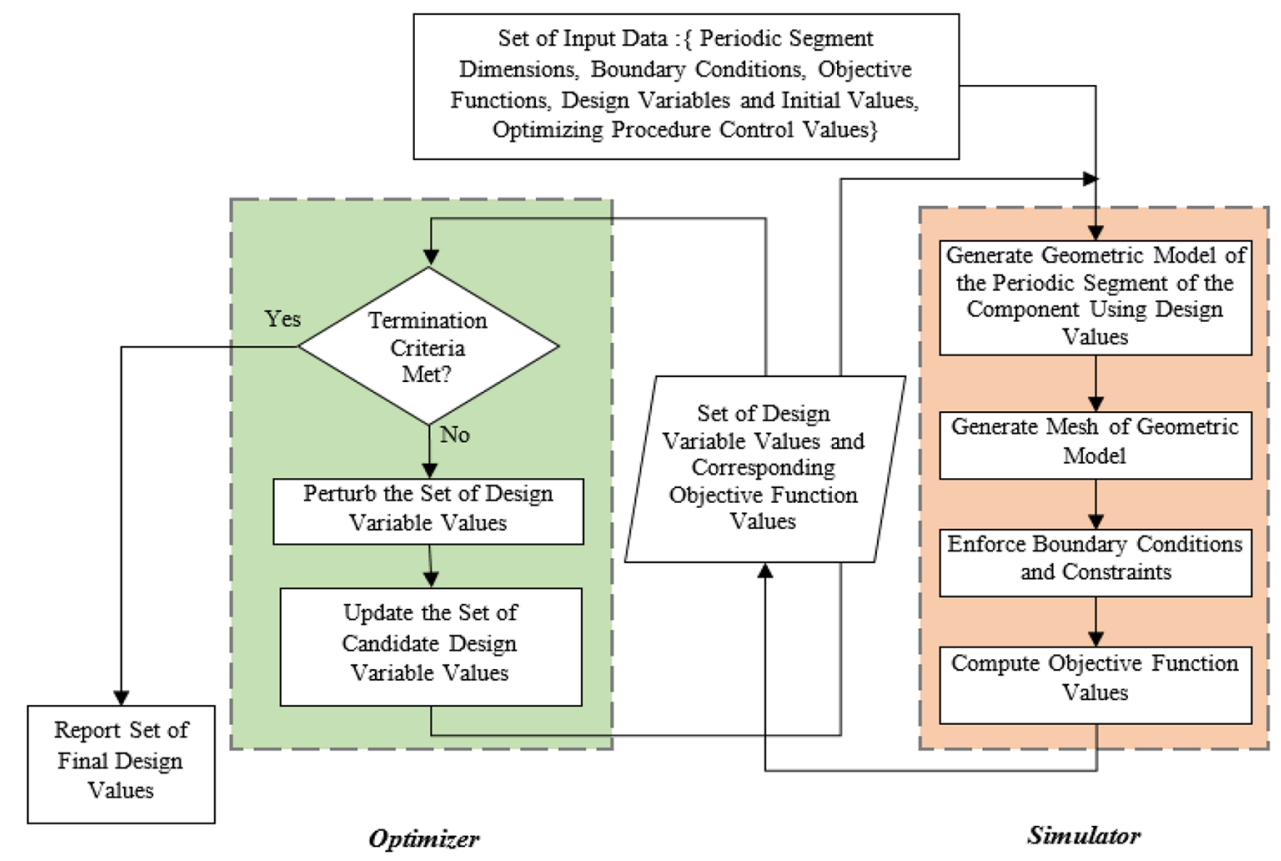

Fig. 5. Illustration of the framework developed for multi-objective optimization of mechanical component [14].
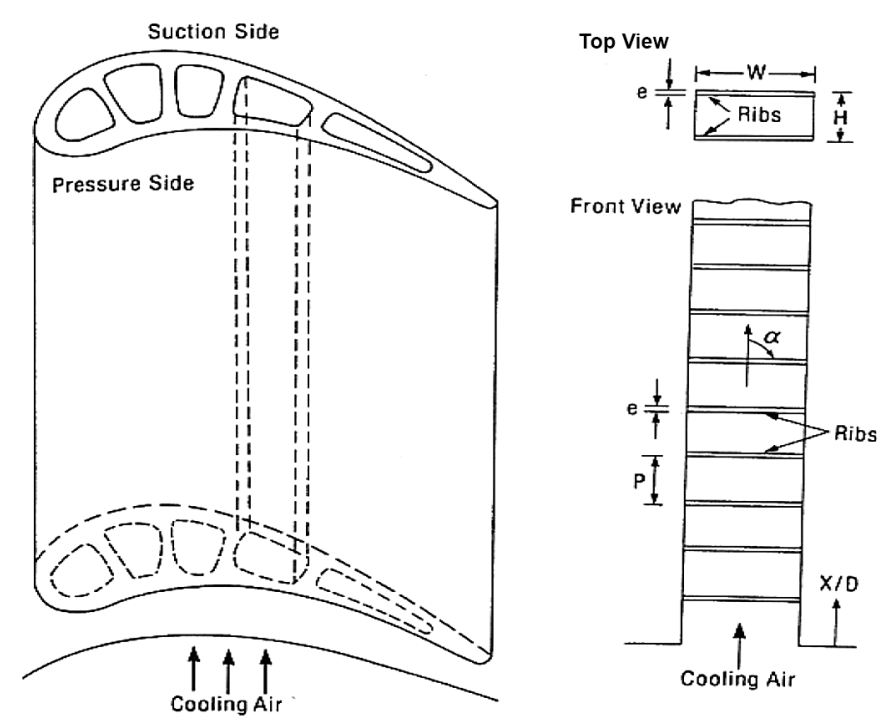

Fig. 6. Series of cooling channels in the gas turbine blade and internal ribs arrangement.

periodic section of the blade-cooling channel by numerical simulation. To calculate the solution for three objective functions, four steps are carried out:

- build computational geometric model of two-dimensional (2D) periodic section of the blade cooling channel utilizing design variable values;

- create the finite element mesh of the model;

- apply the initial and boundary conditions to the computational model;

- solve heat transfer and fluid flow governing equations to obtain the objective functions values.

\subsection{Optimizer component}

The exponential increase in computational power and a significant decrease in hardware cost in recent times resulted in a steep upward trend of using Evolutionary algorithms (EAs) to solve multi-objective problems for optimization [15]. EAs are known to use population-based techniques in multi-objective optimization algorithms which identify multiple Pareto optimal solutions at the end of the final iteration or generation. Optimization procedure begins with a randomly generated initial population of size $\mathrm{N}$ solutions. Next, each solution from population $\mathrm{N}$ is represented by binary strings and every string is the representation of design variable values. Individual strings in the population are assessed with utilizing the simulator to solve for the corresponding fitness values (objective function values). At each generation, a new population is generated using three genetic operators: selection, crossover, and mutation. Individual within the new population is then sent to the simulation section for analysis of objective function values. This process continues iteratively until a user defined termination criterion is fulfilled (number of iterations also called generations). The elitist Non-dominated sorting genetic algorithm II (NSGA II) introduced by Deb et al. [16], is among the popular EAs utilized to solve real-world and conflicting multi-objective design optimization problems. The few salient features of NSGA II are its quick elitist sorting technique that entails a joint pool of a population of the parent and child also delivers a diverse population by applying autonomous crowding distance process. The NSGA II uses elitism by comparing the present candidate solutions in the population using the previously identified finest non-dominated solutions. NSGA II, the selection method utilizes two procedures based on: 


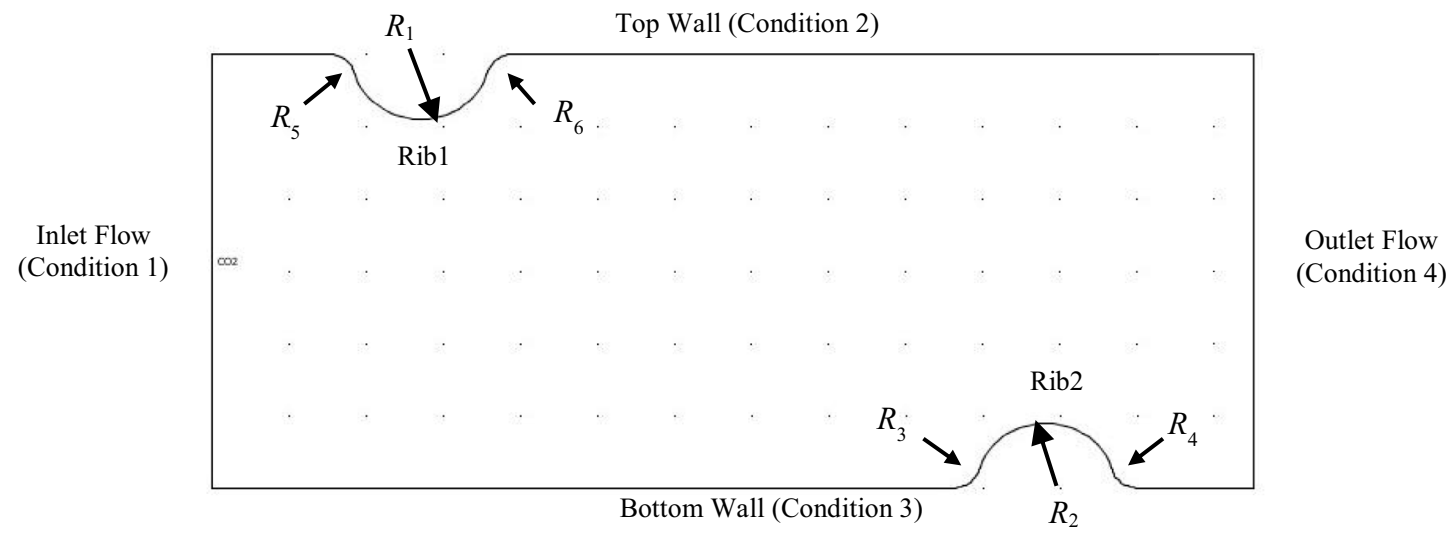

Fig. 7. Periodic section of cooling passage with design variables $R_{1}, R_{2}, R_{3}, R_{4}, R_{5}$, and $R_{6}$.

- ranking by non-domination:

- assignment of crowding distance.

A more comprehensive description of Evolutionary algorithms and NSGA II is well presented in Deb et al. [16-18].

\subsubsection{Genetic operator: selection (or reproduction) operator}

The most important function of the selection operator would be to carry over the better performing candidate solutions (population) and drop the bad performing candidate solutions from the population while keeping the population size. This can be accomplished by carrying out these steps:

- identify the better performing candidate solutions in a population according to their fitness values (select at least $50 \%$ better performing candidate solutions);

- discard poor performing candidate solutions from the population;

- make copies of the better-performing candidate solutions to maintain the initial population size and also to create the mating pool.

\subsubsection{Genetic operator: crossover}

A crossover genetic operator is also known as the recombination operator can be applied to the mating pool of candidate solutions. Crossover operator initiates an exchange of data between chosen better performing solution pairs (called parents) at a particular segment of strings using a probability of crossover $c$. The simulated binary crossover operator (known as SBX) introduced by Deb and Agarwal is applied in this algorithm [19].

\subsubsection{Genetic operator: mutation}

A crossover genetic operator is mainly accountable for intensifying the solution search process and that the mutation operator permits for diversifying the solution search process to avoid the search from getting trapped at a local optimum. After going through the crossover, the newly-created candidate solutions experience a mutation process, where the mutation operator changes a 0 to 1 , and vice versa, using a probability of mutation $m$. The polynomial mutation operator released by Deb and Goyal is applied by NSGA II in which the probability distribution is polynomial [20].

\section{Results and discussions}

The solution evaluation is carried out with a commercial numerical multiphysics simulation program COMSOL. Additionally, the evolutionary algorithm called Nondominated sorting genetic algorithm II (NSGA II) [16] is used as the optimizer as shown in Figure 5. On the other hand, the implementation and success of the current research study do not necessarily depend on using these particular tools or steps.

\subsection{Selection of design variables and input parameters}

As discussed previously, the six design variables $R_{1}$ and $R_{2}$ (radii of ribs 1 and 2 ) and fillet radii $R_{3}, R_{4}, R_{5}$, and $R_{6}$ are the main focus of this research investigation (Fig. 7). The computational domain selected for simulation in COMSOL is shown in Figure 7. The parameters and design variables utilized both in COMSOL and NSGA II are assigned a range of initial values and are described and outlined below. The numerical value range of the design variables $R_{1}$ through $R_{6}$ are shown in Table 1 . These ranges are approximated and selected based on experimental outcomes by Han et al. [4].

Next, flow physics parameters are applied to mimic the realistic conditions of blade internal cooling channel flow to accurately simulate the phenomenon and characteristics of the fluid flow. Here, the compressed cooling air bled from the gas turbine compressor with turbulent flow and nonisothermal physics is utilized.

Table 2 outlines the properties (density, dynamic viscosity) of air at atmospheric pressure and temperature.

Further, Table 3 outlines the starting boundary conditions applied to solve the heat transfer and fluid flow problem and in turn help calculate objective functions using the COMSOL simulation. To create realistic 
Table 1. Design variables range of values (in millimeters).

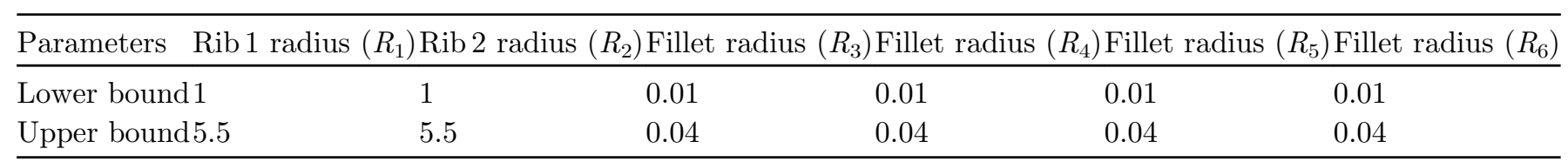

Table 2. Initial conditions of coolant applied for the numerical simulation.

\begin{tabular}{ll}
\hline Fluid & Properties \\
\hline \multirow{2}{*}{ Coolant air } & Density $(\rho)=1.204 \mathrm{~kg} / \mathrm{m}^{3}$ \\
& Dynamic viscosity $\mu=1.983 \times 10^{-5} \mathrm{~kg} / \mathrm{m} \mathrm{s}$ \\
\hline
\end{tabular}

Table 3. Starting boundary conditions applied to heat transfer and fluid flow simulation.

\begin{tabular}{ll}
\hline Boundary & Starting boundary condition \\
\hline Condition 1$)$ & Temperature $(T)=293 \mathrm{~K}$ \\
Inlet-flow & Velocity $(u)=10 \mathrm{~m} / \mathrm{s} ;$ Reynolds \\
& number $\left(R_{e}\right)=20,000$ \\
(Conditions 2 and 3$)$ & Temperature $=393 \mathrm{~K}$ \\
Wall & Thermal wall function \\
(Condition 4$)$ & Convective heat flux and pressure \\
Outlet-flow & $(p)=0$ \\
\hline
\end{tabular}

conditions and to generate turbulence in the flow the cooling passage Inlet Flow (condition 1) is applied a temperature $(T)$ and velocity $(u)$. Fluid velocity is set to zero at wall boundaries (conditions 2 and 3 ), and they are maintained at constant wall temperature, higher than that of the coolant to mimic the phenomenon of heat transfer from blade wall to the coolant. At the Outlet Flow, the convective heat flux and relative pressure are applied (condition 4) indicating fluid departure. The boundary conditions employed are used as starting conditions to solve the heat transfer and fluid flow governing equations iteratively to solve for approximate fluid flow and heat transfer characteristics within the cooling channel.

A pilot study was carried out to study the influence of control parameters of EAs on various problem settings. Table 4 below lists the proposed multi-objective evolutionary algorithm (MOEA) control parameters identified from the pilot study. The evolutionary algorithms work on the principle of iteration, and each iteration is termed as generation and hence the population size, $N$, is a number of candidate design solutions remains constant at every generation. It is identified from the pilot study that, using a small population size can significantly hinder the ability of exploration of the search area and limits the main intention of crossover operations. However, using large population size may increase the search effort but it can be computationally cost prohibitive. For the current feasibility study, a population size of $N=50$ is identified to be appropriate. The maximum number of iterations also
Table 4. NSGA-II input control parameters.

\begin{tabular}{ll}
\hline MOEA parameters & Parameter values \\
\hline Population size $(N)$ & 50 \\
Generations $\left(G_{\max }\right)$ & 100 \\
Selection / Reproduction & $\begin{array}{l}\text { Tournament selection } \\
\text { (rank and crowding distance) }\end{array}$ \\
Probability of crossover & $\mathrm{c}=0.90$ (or, $90 \%)$ \\
Probability of mutation & $\mathrm{m}=0.10$ (or, $10 \%)$ \\
\hline
\end{tabular}

called maximum number of generations, $G e n_{\max }$, denotes the number of generations after which to terminate the MOEA and record the good collection of Pareto optimal solutions (optimal design solutions). It is observed that $G e n_{\max }=100$ generations seem to provide good convergence in the solutions and also to avoid computation burden 100 generations per each optimization study seemed a good fit for this study. In EAs, generation of fresh slightly varied solution (distinct from parents) is obtained by applying the crossover genetic operator. Where, crossover probability, $c$, determine how frequently crossover is carried out in the mating pool. A very low crossover probability reduces the rate of convergence of solution because of reduced exploration rate of all possible solutions. However, a higher probability might result in premature convergence giving rise to the false optimal solution. Generally, the best range of $c$ is between 0.60 and 0.95 and this range is further confirmed from our pilot study. Similarly, the mutation probability, $m$, signifies how frequent sections of an individual candidate solution undergoes arbitrary perturbations. It introduces diversity to the candidate solutions (population) and this probability value ought to be small to prevent the algorithm from turning in to a random search all over the place without proper direction towards the optimal solution. The suggested range for $m$ is between 0 and 0.20 . In the pilot study c $=0.90$ (or, $90 \%$ ) and $\mathrm{m}=0.10$ (or, $10 \%$ ) are proven to have greater convergence rate.

\subsection{Discussion of the results}

As described before, the focus of the study is to construct a multi-objective design optimization framework for mechanical component design, and present at least three conflicting design objectives to concurrently solve and optimize all objectives. The suggested multi-objective optimization framework is successfully formed by combining genetic algorithms and heat transfer and fluid flow simulation tool. For the preliminary test, authors in the beginning, applied the framework onto the single objective 


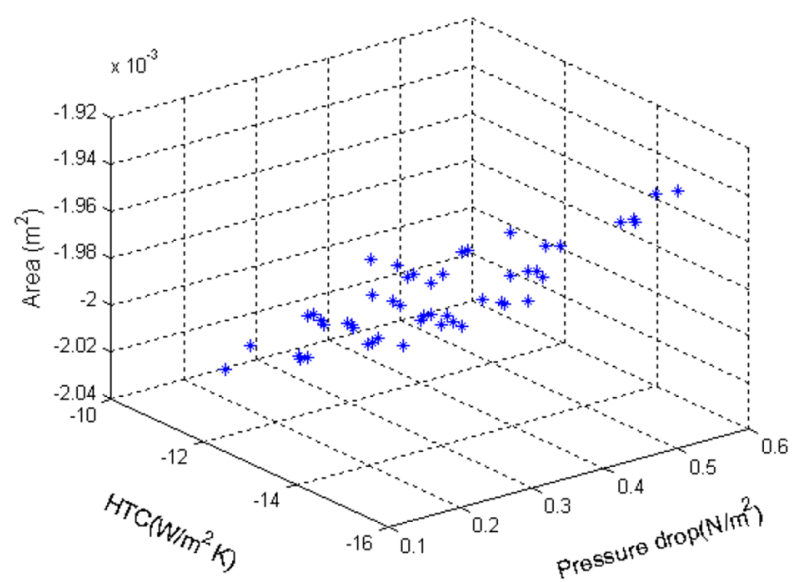

(a) Starting solutions $\left(G e n_{\max }=0\right)$

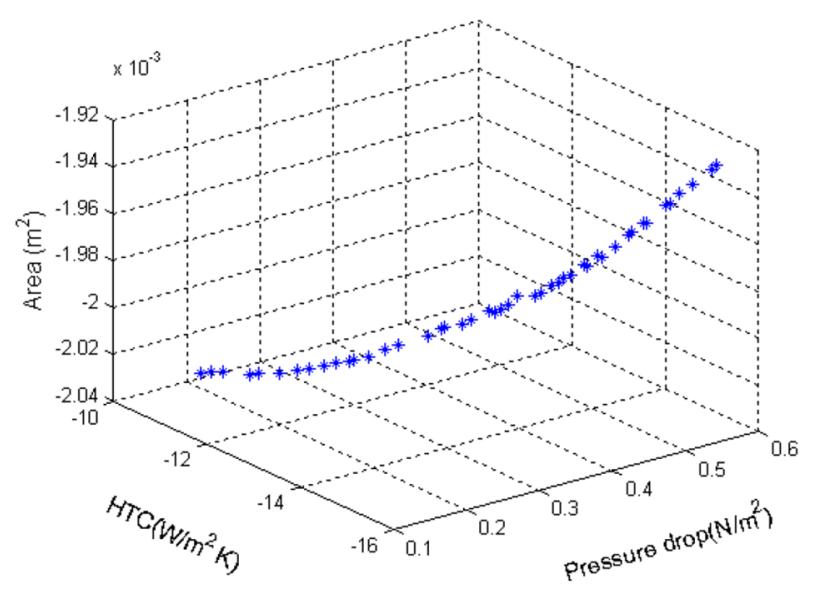

(c) 50 generations $\left(\right.$ Gen $\left._{\max }=50\right)$

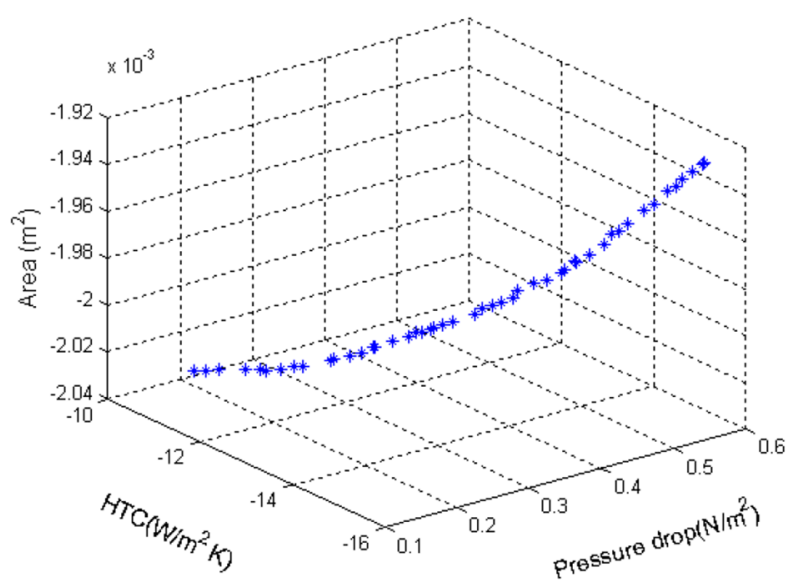

(b) 25 generations $\left(\right.$ Gen $\left._{\max }=25\right)$

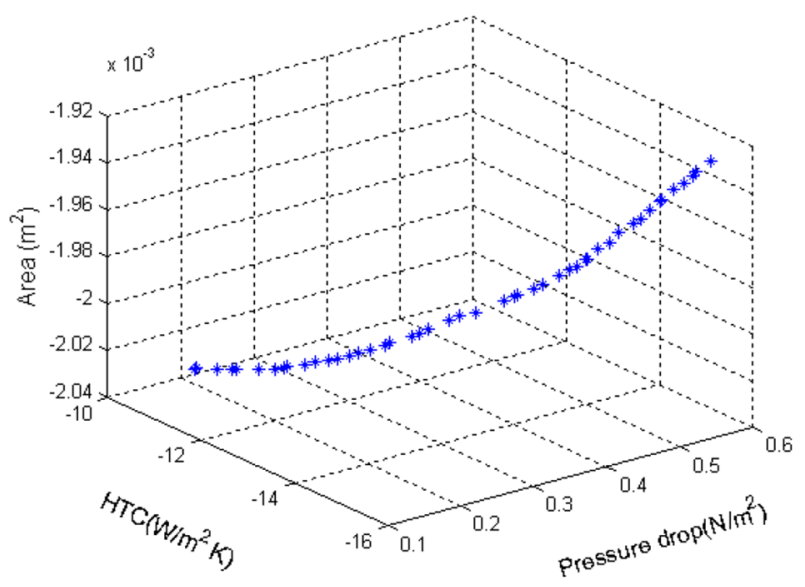

(d) 100 generations $\left(\right.$ Gen $\left.n_{\max }=100\right)$

Fig. 8. Pareto optimal front of 3 objectives and 2 design variables.

and two-objective optimization problem successfully [14], and at present introducing the third objective and its results are presented here. The optimization results obtained by addition of the 3rdobjective function to reduce the expensive blade material utilization by maximizing the blade cavity area $(A)$ within the cooling passage is discussed and shared in this section. It can be observed from the outcome presented that the multiobjective problem at hand is modified to minimization problem, though the two objectives heat transfer coefficient $(h)$ and area $(A)$ are to be maximized. The main reason is, algorithms are in general developed to solve for just one kind of optimization problem, i.e., either maximization or minimization. Open source NSGA utilized within this study is developed to evaluate the minimization problems if applied without modification. The maximization problems can be solved using the duality principle [21], i.e., the problem is changed to a minimization problem by multiplying the objective function by -1 . The duality principle allows one to use conflicting objectives where some objectives need to be minimized and some are to be maximized. Therefore, in our case, the objective functions "heat transfer coefficient $(h)$ " and "area $(A)$ " are multiplied by -1 to modify the multi-objective optimization problem to minimization problem.

The three-objective optimization simulation experiments are carried out using EA control parameters are also utilized on the single and two-objective optimization are shown in Table 4 and further elaborately discussed by Nagaiah et al. [14] (i.e., $P o p=50, G n_{\max }=100, c=90 \%$ and $m=10 \%$ ). Additionally, optimization is performed in steps first by introducing 2 variables $\left(R_{1}\right.$ and $\left.R_{2}\right)$, second by introducing 4 variables $\left(R_{1}, R_{2}, R_{3}\right.$ and $\left.R_{4}\right)$ and third by introducing 6 variables $\left(R_{1}, R_{2}, \ldots R_{6}\right)$ to study the convergence phenomenon. It has been noticed from three-step experimental runs, the introduction of more 


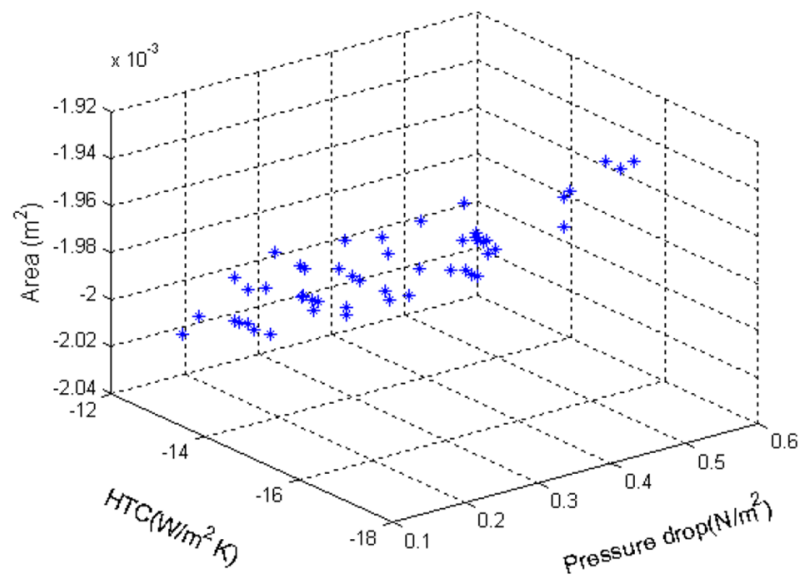

(a) Starting solutions $\left(\mathrm{Gen}_{\max }=0\right)$

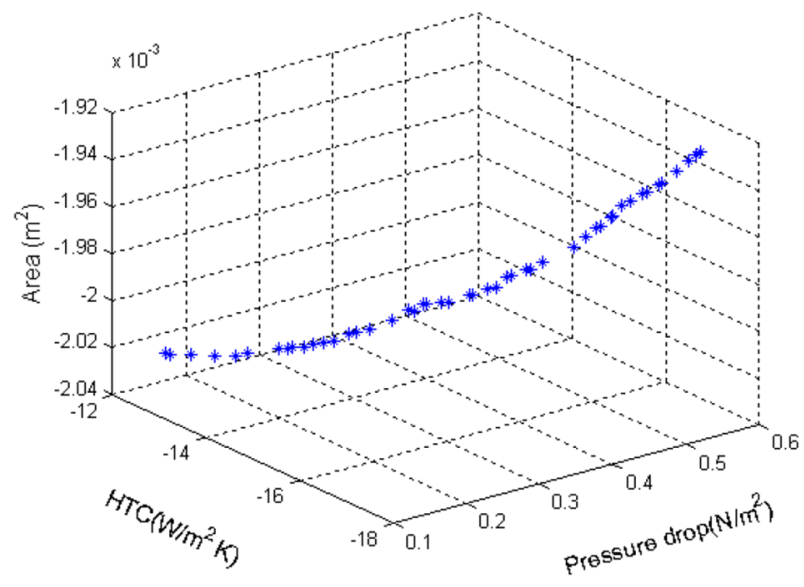

(c) 50 generations $\left(\mathrm{Gen}_{\max }=50\right)$

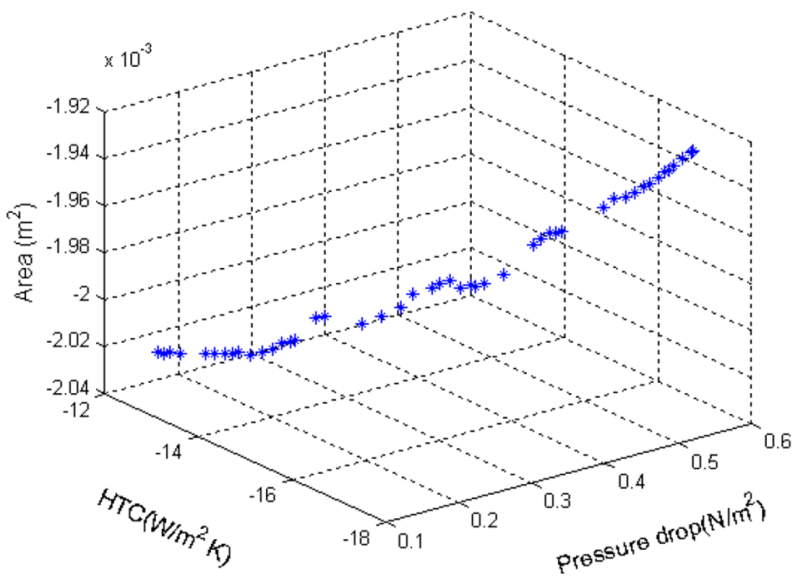

(b) 25 generations $\left(\right.$ Gen $\left._{\max }=25\right)$

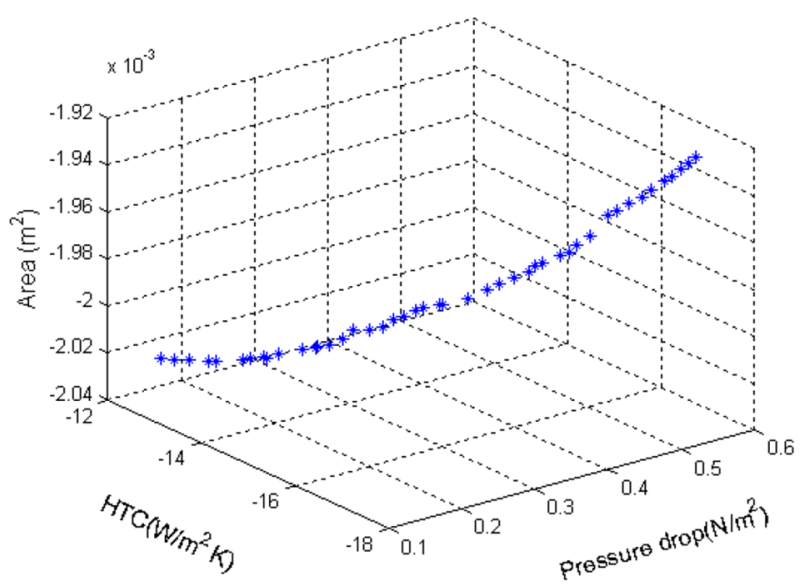

(d) 100 generations $\left(\right.$ Gen $\left._{\text {max }}=100\right)$

Fig. 9. Pareto optimal front of 3 objectives and 4 design variables.

design variables into the problem caused a decrease in the convergence of the solution towards Pareto optimal front (Figs. 8-10 ). From the observation, it is evident that the additional number of variables made the solution space to increase drastically and required more search effort (iterations) and it demands the selection of appropriate genetic algorithm control parameters. Figure 10 shows the final graphical representation of all 3 objective functions and 6 design variable Pareto optimal front at different generation intervals. The $x$-axis represents the objective function cooling air pressure drop $(\Delta p)$, which is to be minimized. The $y$-axis represents an objective function, heat transfer coefficient $(h)$, which is to be maximized. The $z$-axis represents objective function of the area $(A)$, which is again to be maximized.

The graphical representation in Figure 10 (a) shows the set of starting objective function values computed before the application of multi-objective optimization. Figure 10 (b), (c) and (d) present solutions converging and progression towards Pareto optimal front after computation of 25, 50 and 100 generations, respectively. To illustrate in detail the significance of the final Pareto optimal solutions, in Figure 1 (b), three objective function solution values are selected from the Pareto front from both ends and at the middle (as shown in Fig. 11(b)) and their corresponding design variables value as shown in the table in Figure 11 (a). These design variabled represent three designs of cooling passage ribs as shown in Figure 1. It can be noticed that design 1 includes smaller rib radii $\left(R_{1}=1 \mathrm{~mm}\right.$ and $R_{2}=1 \mathrm{~mm}$ ) leading to a reduced heat transfer coefficient $h$ $\left(11.09 \mathrm{~W} / \mathrm{m}^{2} \mathrm{~K}\right)$, reduced pressure drop $\Delta p\left(0.1485 \mathrm{~N} / \mathrm{m}^{2}\right)$, and increased cavity area $A\left(0.002022 \mathrm{~m}^{2}\right)$. Similarly, on the other extreme end, design 3 has larger ribs $\left(R_{1}=5.49 \mathrm{~mm}\right.$ 


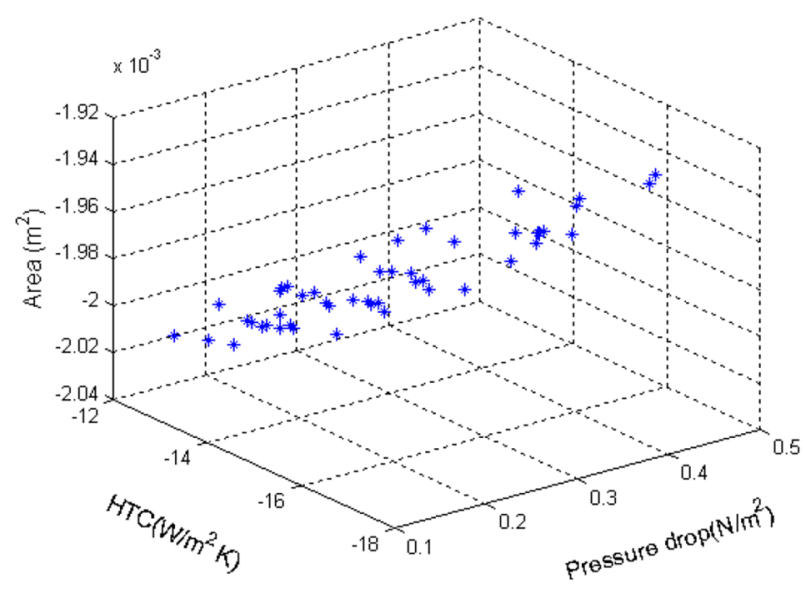

(a) Starting solutions $\left(\right.$ Gen $\left.n_{\max }=0\right)$

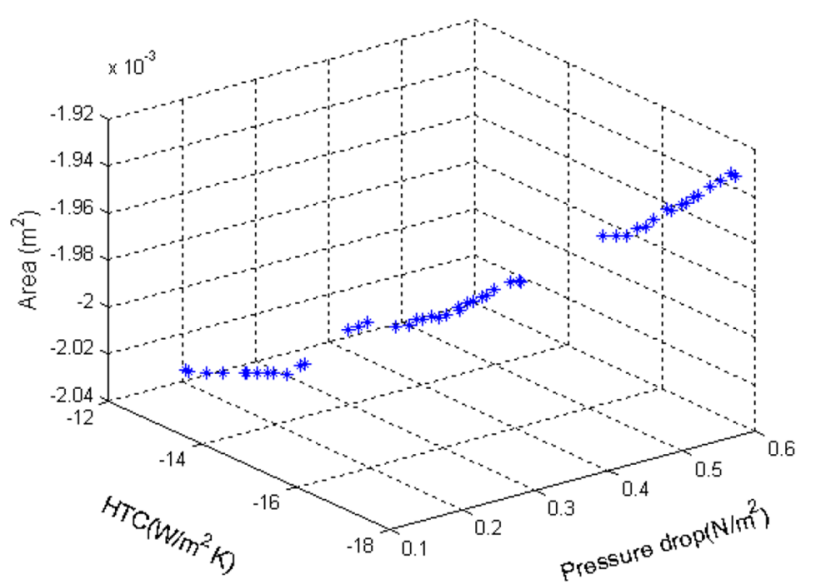

(c) 50 generations $\left(G e n_{\text {max }}=50\right)$

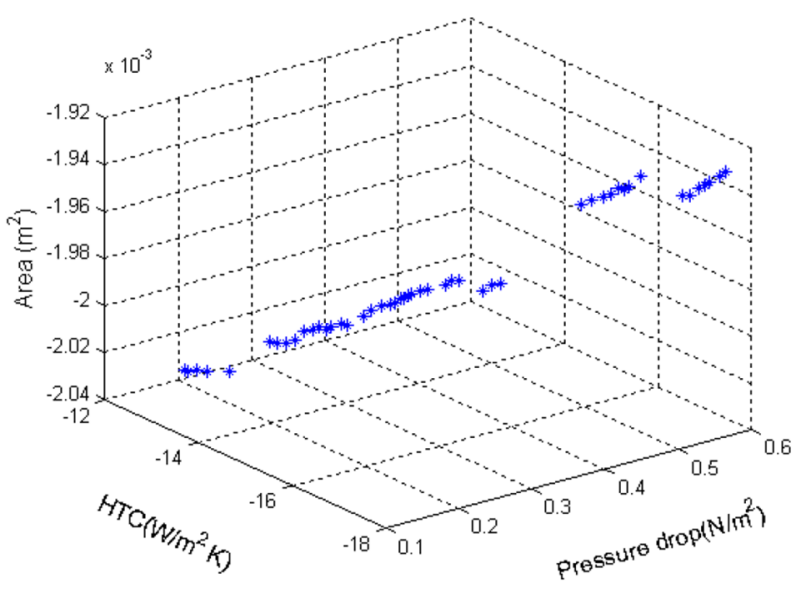

(b) 25 generations $\left(\right.$ Gen $\left.n_{\text {max }}=25\right)$

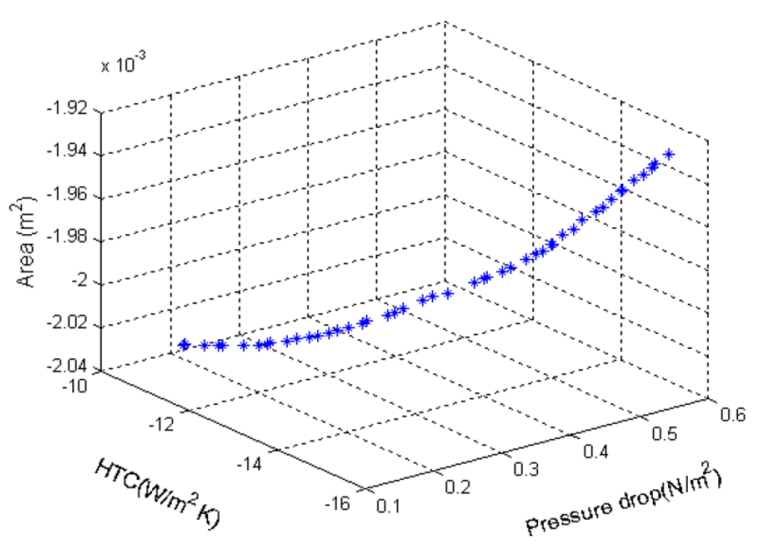

(d) 100 generations $\left(\right.$ Gen $\left._{\max }=100\right)$

Fig. 10. Pareto optimal front of 3 objectives and 6 design variables.

and $\left.R_{2}=5.49 \mathrm{~mm}\right)$ resulting in increased heat transfer coefficient $h\left(15.43 \mathrm{~W} / \mathrm{m}^{2} \mathrm{~K}\right)$, increased pressure drop $\Delta p$ $\left(0.5789 \mathrm{~N} / \mathrm{m}^{2}\right)$ and reduced cavity area $A\left(0.00193 \mathrm{~m}^{2}\right)$. Lastly, design 2 is chosen from the middle of the Pareto front with one larger and other smaller rib $\left(R_{1}=1.025 \mathrm{~mm}\right.$ and $R_{2}=4.936 \mathrm{~mm}$ ) which results in moderate heat transfer coefficient $h\left(14.15 \mathrm{~W} / \mathrm{m}^{2} \mathrm{~K}\right)$, pressure drop $\left(0.2955 \mathrm{~N} / \mathrm{m}^{2}\right)$ and cavity area $A\left(0.001985 \mathrm{~m}^{2}\right)$. These results further demonstrate the significance of the multi-objective optimization framework developed. It is evident from our framework that, one can solve for thousands of designs in an automated technique and select best designs based on objective function values or design variable values if there is a constraint on design variable values.

The Pareto optimal front solutions can be further split into clusters to accommodate three design types and also to ease the design selection process for the designer who is at the dilemma of choosing an appropriate design to fulfill the particular requirements (Fig. 2). The clustering technique can be demonstrated as shown below in Figure 12 by forming a group of three types of designs. Subset 1 (cluster) comprises of best solutions which meet minimization of pressure drop $(\Delta p)$ and maximization of cavity area $(A)$ objectives better compared to any other solutions in Subset 2 and 3 . In the same way, Subset 3 meets the maximization of heat transfer coefficient $(h)$ objective better compared to any other solutions in the Subsets 1 and 2. Optimal solutions in Subset 2 are between Subsets 1 and 3 with moderate optimization of all three objective functions. Therefore, clustering gives a quick visual comprehension into the solutions and helps the designer in choosing solution according to his preferences for objective function values. 


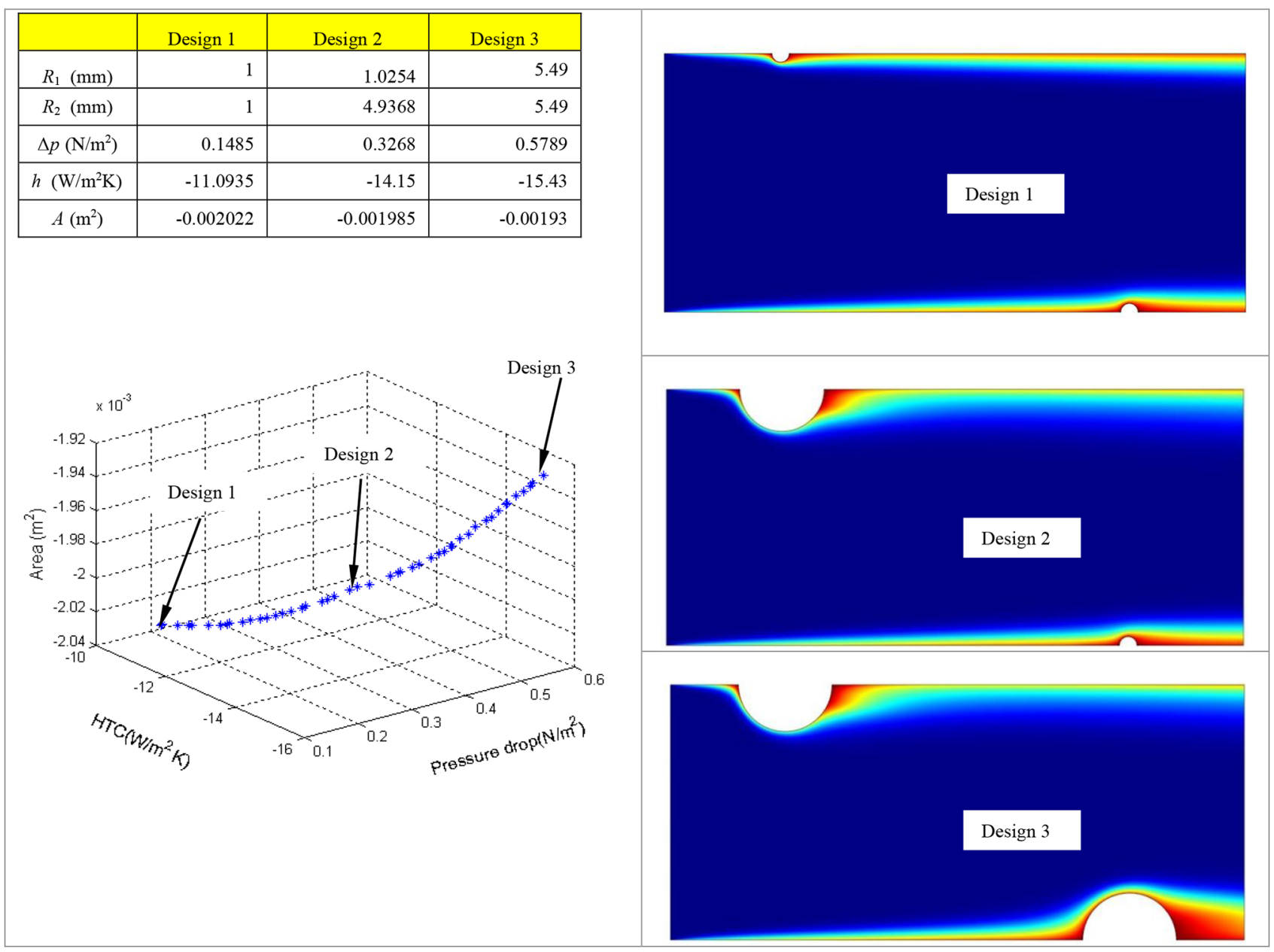

Fig. 11. Illustration of the design of cooling passage for 3 distinct optimal solutions.

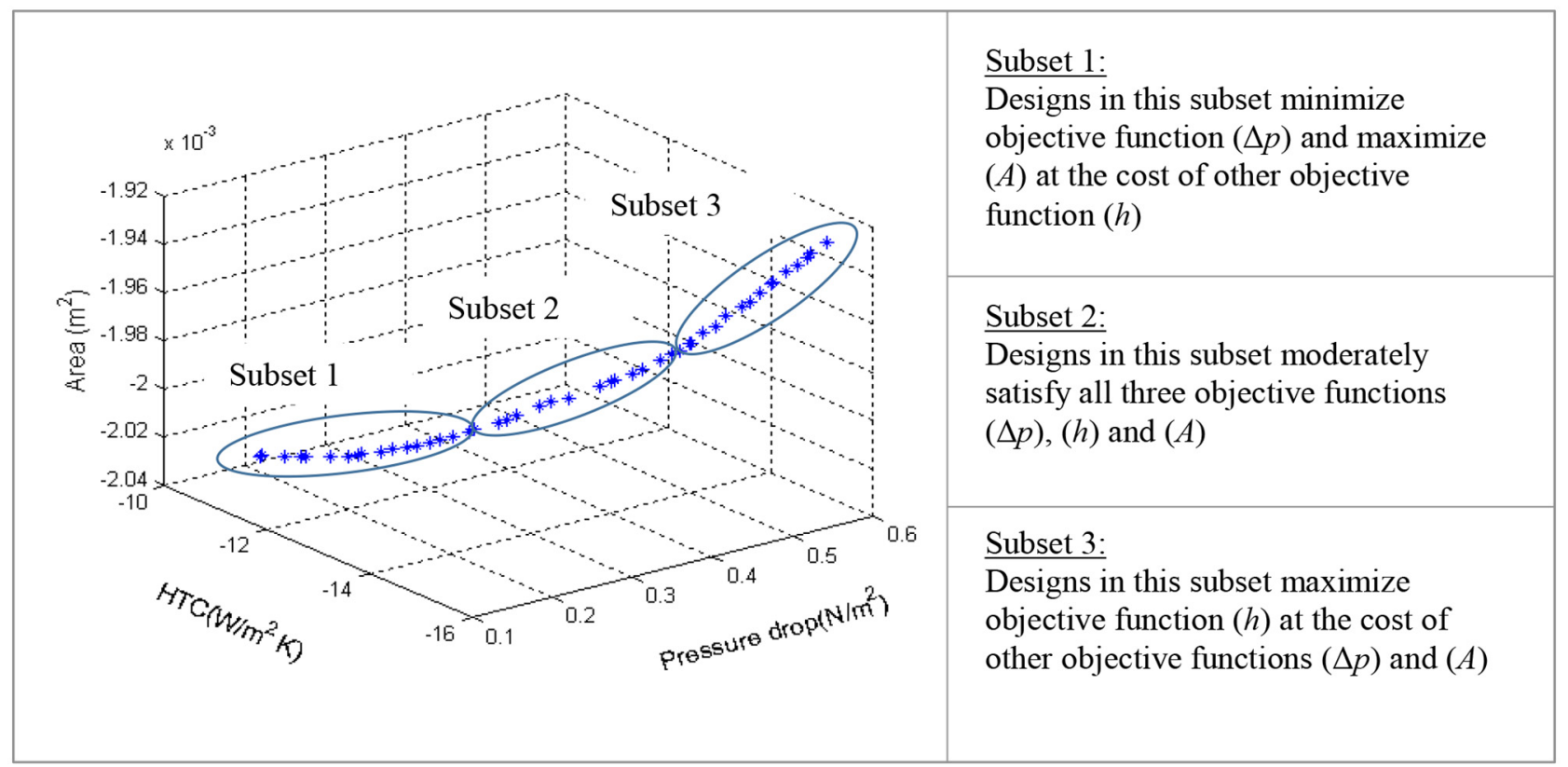

Fig. 12. Pareto optimal solution front separated into three subsets (clusters) of solutions. 


\section{Conclusion}

The proposed framework is comprising, evolutionary algorithm NSGA-II and heat transfer and fluid flow simulation program COMSOL is successfully integrated and implemented to optimization of an industrial gas turbine blade cooling passage design configuration. The optimization is carried out in an automated manner to a final single best solution through maximization of heat transfer coefficient $(h)$ in single objective optimization. Following the introduction of the second objective function resulted in the experimental outcome of non-dominated Pareto optimal solutions of maximization of heat transfer coefficient $(h)$ and minimization of pressure drop $(\Delta p)$ for two objective optimizations. Lastly, the introduction of third objective function provided non-dominated Pareto optimal solutions for maximization of heat $(h)$, minimization of $(\Delta p)$ and maximization cooling passage cavity area $(A)$ for three objective optimizations.

The experimental results shed more lights and provided insight into understanding the physics of design by demonstrating the correlation between design variables and objective functions. The proposed automated optimization framework can be regarded as a stepping stone and supporting tool at the beginning of any complex design problem with conflicting objective functions. It is the authors' belief that the concept and framework developed in this research will help others to implement and go beyond simple component design and apply this technique to more complex design problems.

\section{Nomenclature}

$\begin{array}{ll}\varphi & \text { Effectiveness of cooling } \\ \dot{m} & \text { Rate of mass-flow rate }(\mathrm{kg} / \mathrm{s}) \\ u & \text { Velocity of flow }(\mathrm{m} / \mathrm{s}) \\ A & \text { Area of cooling channel under consideration } \\ & \left(\mathrm{m}^{2}\right) \\ R_{1} \text { and } R_{2} & \text { Radii (ribs } 1 \text { and } 2) \\ R_{3} \ldots R_{6} & \text { Fillets radii (ribs } 1 \text { and } 2) \\ N & \text { Size of the population } \\ c & \text { Probability of crossover } \\ m & \text { Probability of mutation }\end{array}$

\section{References}

1. N.R. Nagaiah, C.D. Geiger, Evolutionary numerical simulation approach for design optimization of gas turbine blade cooling channels, Int. J. Simulat. Multidiscipl. Des. Optim. 5, A22 (2014)

2. M.P. Boyce, Gas turbine engineering handbook (Gulf Professional Publications, Boston, 2006), Vol. 3, pp. 936
3. H. Moustapha, Axial and radial turbines (Concepts ETI, Inc., 2003), pp. 358

4. J.C. Han, S. Dutta, S. Ekkad, Gas turbine heat transfer and cooling technology (Taylor \& Francis, New York, 2000), pp. 646

5. J.C. Han, Recent studies in turbine blade cooling, Int. J. Rotating Mach. 10, 443-457 (2004)

6. E. Logan, Handbook of turbomachinery. Mechanical engineering (Marcel Dekker, Inc., New York, 1995), Vol. 93, pp. 471

7. R.J. Goldstein, Heat transfer in gas turbine systems. Annals of the New York Academy of Sciences 007r7 -8923 (New York Academy of Sciences, New York, 2001), Vol. 934, pp. 522

8. L.M. Gosselin, Tye-Gingras, F. Mathieu-Potvin, Review of utilization of genetic algorithms in heat transfer problems, Int. J. Heat Mass Transfer 52(9-10), 2169-2188 (2009)

9. P.M. Ligrani, M.M. Oliveira, T. Blaskovich, Comparison of heat transfer augmentation techniques, AIAA J. 41, 337-362 (2003)

10. K.Y. Kim, H.M. Kim, Shape optimization of rib-roughened surface to enhance turbulent heat transfer, Int. J. Heat Mass Transfer 45, 2719-2727 (2002)

11. H.M. Kim, K.Y. Kim, Design optimization of rib-roughened channel to enhance turbulent heat transfer, Int. J. Heat Mass Transfer 47(23), 5159-5168 (2004)

12. K.Y. Kim, Y.M. Lee, Design optimization of internal cooling passage with V-shaped ribs, Num. Heat Transfer Part A: Appl. Int. J. Comput. Methodol. 51, 1103-1118 (2007)

13. K.Y. Kim, H.M. Kim, Optimization of the three-dimensional angled ribs with RANS analysis of turbulent heat transfer (ASME Turbo Expo, Vienna, Austria, 2004)

14. N.R. Nagaiah, C.D. Geiger, J.S. Kapat, Three-objective optimization for the design of mechanical component using evolutionary numerical simulation approach, 52nd AIAA, SAE/ASEE Joint Propulsion Conference proceedings, Salt Lake City, Utah 2016, p. 4959

15. K. Deb, Current trends in evolutionary multi-objective optimization, Int. J. Simul. Multidiscipl. Des. Optim. 1(1), 1-8 (2007)

16. K. Deb, et al., A fast elitist non-dominated sorting geneticalgorithm for multi-objective optimization: NSGA-II, IEEE Trans. 6, 197 (2002)

17. N. Srinivas, K. Deb, Multiobjective optimization using nondominated sorting in genetic algorithms, Int. J. Evolut. Comput. 2(3), 221-248 (1994)

18. K. Deb, Multi-objective optimization using evolutionary algorithms. Wiley-Interscience series in systems and optimization (John Wiley \& Sons, New York, 2001), Vol. 1, pp. 497

19. K. Deb, R.B. Agarwal, Simulated binary crossover for continuous search space, Complex Syst. 9, 115-148 (1995)

20. K. Deb, M. Goyal, A combined genetic adaptive search (GeneAS) for engineering design, Comput. Sci. Inform. 26 (4), 30-45 (1996)

21. S.S. Rao, Optimization: theory and applications (Halsted Press, 1984), Vol. 2, 2nd ed., pp. 747

Cite this article as: Narasimha R. Nagaiah, Christopher D. Geiger, Application of evolutionary algorithms to optimize cooling channels, Int. J. Simul. Multidisci. Des. Optim. 10, A4 (2019) 